\title{
Lossless compression of digital mammography using base switching method
}

\author{
Ravi kumar Mulemajalu1, Shivaprakash Koliwad ${ }^{2}$
}

${ }^{1}$ Corresponding author, Department of IS\&E., KVGCE, Sullia, Karnataka, India; ${ }^{2}$ Department of E\&C., MCE, Hassan, Karnataka, India. Email: ${ }^{1}$ perajeravi@yahoo.com, ${ }^{2}$ spksagar2006@yahoo.co.in

Received 31March 2009; revised 15 May 2009; accepted 25 May 2009.

\begin{abstract}
Mammography is a specific type of imaging that uses low-dose x-ray system to examine breasts. This is an efficient means of early detection of breast cancer. Archiving and retaining these data for at least three years is expensive, difficult and requires sophisticated data compression techniques. We propose a lossless compression method that makes use of the smoothness property of the images. In the first step, de-correlation of the given image is done using two efficient predictors. The two residue images are partitioned into non overlapping sub-images of size $4 \times 4$. At every instant one of the sub-images is selected and sent for coding. The sub-images with all zero pixels are identified using one bit code. The remaining subimages are coded by using base switching method. Special techniques are used to save the overhead information. Experimental results indicate an average compression ratio of 6.44 for the selected database.
\end{abstract}

Keywords: Lossless Compression; Mammography image; Prediction; Storage Space

\section{INTRODUCTION}

Breast cancer is the most frequent cancer in the women worldwide with 1.05 million new cases every year and represents over $20 \%$ of all malignancies among female. In India, 80,000 women were affected by breast cancer in 2002. In the US, alone in 2002 , more than 40,000 women died of breast cancer. $98 \%$ of women survive breast cancer if the tumor is smaller than $2 \mathrm{~cm}$ [1]. One of the effective methods of early diagnosis of this type of cancer is non-palpable, non-invasive mammography. Through mammogram analysis radiologists have a detection rate of $76 \%$ to $94 \%$, which is considerably higher than $57 \%$ to $70 \%$ detection rate for a clinical breast ex- amination [2].

Mammography is a low dose x-ray technique to acquire an image of the breast. Digital image format is required in computer aided diagnosis (CAD) schemes to assist the radiologists in the detection of radiological features that could point to different pathologies. However, the usefulness of the CAD technique mainly depends on two parameters of importance: the spatial and grey level resolutions. They must provide a diagnostic accuracy in digital images equivalent to that of conventional films. Both pixel size and pixel depth are factors that critically affect the visibility of small low contrast objects or signals, which often are relevant information for diagnosis [3]. Therefore, digital image recording systems for medical imaging must provide high spatial resolution and high contrast sensitivity. Due to this, mammography images commonly have a spatial resolution of 1024x1024, 2048x2048 or 4096x4096 and use 16, 12 or 8 bits/pixel. Figure 1 shows a mammography image of size $1024 \times 1024$ which uses 8 bits/pixel.

Nevertheless, this requirement retards the implementation of digital technologies due to the increment in processing and transmission time, storage capacity and cost that good digital image quality implies. A typical mammogram digitized at a resolution of 4000x 5000 pixels with $50-\mu \mathrm{m}$ spot size and 12 bits results in approximately $40 \mathrm{Mb}$ of digital data. Processing or transmission time of such digital images could be quite long. An efficient data compression scheme to reduce the digital data is needed.

The goal of the image compression techniques is to represent an image with as few bits as possible in such a way that the original image can be reconstructed from this representation without or with minimum error or distortion. Basically image compression techniques have been classified into two categories namely lossy and lossless methods. Lossy compression methods cannot achieve exact recovery of the original image, but achieves significant compression ratio. Lossless compression techniques, as their name implies, involve no loss of information. The original data can be recovered 


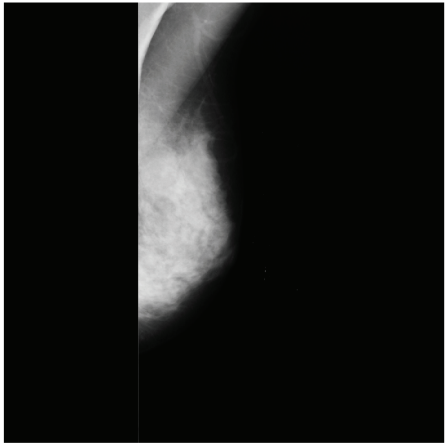

Figure 1. A Mammography image of size $1024 \times 1024$ which uses 8 bits/pixel.

exactly from the compressed data. In medical applications, lossless coding methods are required since loss of any information is usually unacceptable [4]. Performance of the lossless compression techniques can be measured in terms of their compression ratio, bits per pixels required in the compressed image and the time for encoding and decoding. On the other hand, since the lossy compression techniques discard some information, performance measure includes the mean square error and peak signal to noise ratio (PSNR) in addition to the measures used for the lossless compression.

Lossless image compression systems typically function in two stages [5]. In the first stage, two-dimensional spatial redundancies are removed by using an image model which can range from a relatively simple causal prediction used in the JPEG-LS $[6,7]$ standard to a more complicated multi-scale segmentation based scheme. In the second stage, the two-dimensional de-correlated residual which is obtained from the first stage, along with any parameters used to generate the residual is coded with a one-dimensional entropy coder such as the Huffman or the Arithmetic coder.

Existing lossless image compression algorithms can be broadly classified into two kinds: Those based on prediction and those that are transform based. The predictive coding system consists of a prediction that at each pixel of the input image generates the anticipated value of that pixel based on some of the past pixels and the prediction error is entropy coded. Various local, global and adaptive methods can be used to generate prediction. In most cases, however the prediction is formed by a linear combination of some previous pixels. The variance of the prediction error is much smaller than the variance of the gray levels in the original image. Moreover, the first order estimate of the entropy of the error image is much smaller than the corresponding estimate for the original image. Thus higher compression ratio can be achieved by entropy coding the error image. The past pixels used in the prediction are collectively referred to as a context. The popular JPEG-LS standard uses the prediction based coding technique [8]. Trans- form based algorithms, on the other hand, are often used to produce a hierarchical or multi-resolution representation of an image and work in the frequency domain. The popular JPEG-2000 standard uses the transform based coding technique [9].

Several techniques have been proposed for the lossless compression of the digital Mammography. A. Neekabadi et al. [10] uses chronological sifting of prediction errors and coding the errors using arithmetic coding. For the 50 MIAS (Mammography Image Analysis Society) images, CSPE gives better average compression ratio than JPEG-LS and JPEG-2000. Xiaoli Li et al. [11] uses grammar codes in that the original image is first transformed into a context free grammar from which the original data sequence can be fully reconstructed by performing parallel and recursive substitutions and then using an arithmetic coding algorithm to compress the context free grammar. Compression ratio achieved is promising but it involves more complicated processing and large computation time. Delaunay triangulation method [12] is another approach. It uses geometric predictor based on irregular sampling and the Delaunay triangulation. The difference between the original and the predicted is calculated and coded using the JPEG-LS approach. The method offers lower bit rate than the JPEG-LS, JPEG-lossless, JPEG2000 and PNG. A limitation is the slow execution time. Lossless JPEG2000 and JPEG-LS are considered as the best methods for the mammography images. Lossless JPEG 2000 methods are preferred due to the wide variety of features, but are suffered from a slightly longer encoding and decoding time [13].

Recently, there have been a few instances of using segmentation for lossless compression. Shen and Rangayyan [14] proposed a simple region growing scheme which generates an adaptive scanning pattern. A difference image is then computed and coded using the JBIG compression scheme. Higher compression ratio is possible with such a scheme for the high resolution medical images. But the application of the same scheme to normal images did not result in significant performance improvement. Another scheme reported in literature involves using a variable block size segmentation(VBSS) to obtain a context sensitive encoding of wavelet coefficients, the residual being coded using a Huffman or Arithmetic coder $[15,16]$. The performance of the method is comparable to that of the lossless JPEG standard. Mar wan Y. et al. [17] proposed fixed block based (FBB) lossless compression methods for the digital mammography. The algorithm codes blocks of pixels within the image that contain the same intensity value, thus reducing the size of the image substantially while encoding the image at the same time. FBB method alone gives small compression ratio but when used in conjunction with LZW it provides better compression 
ratio.

We propose a method based on Base switching (BS). Trees-Juen Chuang et al. [18] have used Base-switching method to compress the general images. [19] And [20] also have used the same concept for the compression of digital images. The algorithm segments the image into non overlapping fixed blocks of size $\mathrm{n} \times \mathrm{n}$ and codes the pixels of the blocks based on the amount of smoothness. In the proposed work we have optimized the original BS method for the compression of mammography images. Specific characteristics of mammography images are well suited for the proposed method. These characteristics include low number of edges and vast smooth regions.

The organization of the paper is as follows. Section 2 describes the basic Base Switching (BS) method. The proposed algorithm is given in Section 3. Experimental results and conclusion are given in Sections 4 and 5 respectively.

\section{BASE-SWITCHING ALGORITHM}

The BS method divides the original image (gray-level data) into non-overlapping sub-images of size $n \times n$. Given a $n \times n$ sub-image A, whose $\mathrm{N}$ gray values are $\mathrm{g}_{0}, \mathrm{~g}_{1}, \ldots \mathrm{g}_{\mathrm{N}-1}$, define the "minimum" $\mathrm{m}$, "base" $\mathrm{b}$ and the "modified sub-image " $\mathrm{A}$, whose $\mathrm{N}$ gray values are $\mathrm{g}_{0}{ }^{\mathrm{I}}, \mathrm{g}_{1}{ }_{1}^{\mathrm{I}}, \ldots \mathrm{g}_{\mathrm{N}-1}^{\mathrm{I}}$, by

$$
\begin{gathered}
m=\min g_{i} \\
b=\max g_{i}-\min g_{i}+1 \\
A_{n \times n}^{I}=A_{n \times n}-m \times I_{n \times n} \\
\text { Also, } g_{i}{ }^{I}=g_{i}-m \quad \text { for all } \mathrm{i}=0 \text { to } \mathrm{N}-1
\end{gathered}
$$

where $\mathrm{N}=n \times n$ and each of the elements of $\mathrm{I}$ is 1 . The value of ' $b$ ' is related to smoothness of the sub-image where smoothness is measured as the difference between maximum and minimum pixel values in the sub-image.

The number of bits required to code the gray values $\mathrm{g}_{\mathrm{i}}{ }^{\mathrm{I}}$ is,

$$
\mathrm{B}=\left\lceil\log _{2} b\right\rceil
$$

Then, total bits required for the whole sub-image is,

$$
Z_{A}=\mathrm{N} \times\left\lceil\log _{2} b\right\rceil \text { bits. }
$$

For example, for the sub-image of Figure 2, $\mathrm{n}=4$, $\mathrm{N}=16, \mathrm{~m}=95 \& \mathrm{~b}=9$. Modified sub-image of Figure 3 is obtained by subtracting 95 from every gray values of $A$.

For the sub-image in Figure 3, since $\mathrm{B}=4, Z_{A}=64$ bits.

\begin{tabular}{|c|c|c|c|}
\hline 95 & 96 & 97 & 99 \\
\hline 96 & 97 & 103 & 103 \\
\hline 97 & 96 & 96 & 103 \\
\hline 96 & 96 & 97 & 103 \\
\hline
\end{tabular}

Figure 2. A sub-image A with $\mathrm{n}=4, \mathrm{~N}=16$, $\mathrm{m}=95, \mathrm{~b}=9 \& \mathrm{~B}=4$.

\begin{tabular}{|l|l|l|l|}
\hline 0 & 1 & 2 & 4 \\
\hline 1 & 2 & 8 & 8 \\
\hline 2 & 1 & 1 & 8 \\
\hline 1 & 1 & 2 & 8 \\
\hline
\end{tabular}

Figure 3. Modified sub-image $\mathrm{A}^{\mathrm{I}}$.

In order to reconstruct $\mathrm{A}$, value of $\mathrm{B}$ and $\mathrm{m}$ should be known. Therefore encoded bit stream consists of $\mathrm{m}$, B and $\mathrm{A}^{\mathrm{I}}$ coded using $\mathrm{B}$ bits. In the computation of $\mathrm{B}$, If $\mathrm{b}$ is not an integer power of $2, \log _{2}(\mathrm{~b})$ is rounded to the next higher integer. Thus, in such cases, higher number of bits is used than absolutely required. BS method uses the following concept to exploit this redundancy.

$$
\text { It is found that, } \min g_{i}{ }^{\mathrm{I}}=0 \text { and } \max g_{i}{ }^{\mathrm{I}}=b-1
$$

The image $A^{\mathrm{I}}=\left(\mathrm{g}_{0}{ }_{1}^{\mathrm{I}}, \mathrm{g}_{1}, \ldots, \mathrm{g}_{\mathrm{N}-1}^{\mathrm{I}}\right)$ can be treated as an $\mathrm{N}$ digit number $\left(\mathrm{g}_{0}{ }^{1} \mathrm{~g}_{1}{ }^{1} \ldots \mathrm{g}_{\mathrm{N}-1}\right)_{b}$ in the base $\mathrm{b}$ number system. An integer value function $\mathrm{f}$ can be defined such that $f\left(A^{\mathrm{I}}, \mathrm{b}\right)=$ decimal integer equivalent to the base- $b$ number.

$$
\begin{gathered}
=\sum_{i=0}^{N-1} g_{i}{ }^{I} \times b^{i} \\
=\mathrm{g}_{0}{ }^{\mathrm{I}}+\mathrm{g}_{1}{ }_{1} \times \mathrm{b}^{\cdots}+\cdots \cdot \mathrm{g}_{\mathrm{N}-1}^{\mathrm{I}} \times \mathrm{b}^{\mathrm{N}-1}
\end{gathered}
$$

\begin{tabular}{|c|c|c|}
\hline $\begin{array}{l}\text { Min. value in } \\
\text { the } \mathrm{n} \times \mathrm{n} \text { block. } \\
\quad(8 \text { bits })\end{array}$ & $\begin{array}{c}\text { Value of } \mathrm{b} \\
\text { For the } \mathrm{n} \times \mathrm{n} \\
\text { block } \\
(8 \text { bits })\end{array}$ & $\begin{array}{c}\mathrm{f}\left(\mathrm{A}^{\mathrm{I}}, \mathrm{b}\right) \text { coded using } \\
Z_{B} \text { bits } \\
\left(Z_{B} \text { bits }\right)\end{array}$ \\
\hline
\end{tabular}

Then, number of bits required to store the integer $f\left(\mathrm{~A}^{\mathrm{I}}\right.$, b) is

$$
Z_{B}=\left\lceil\log _{2} b^{N}\right\rceil
$$

Reconstruction of $\mathrm{A}^{\mathrm{I}}$ is done by switching the binary (base 2) number to a base $b$ number. Therefore, reconstruction of A needs the value of $m$ and $b$. The format of representation of a sub-image is as shown below.

For the example of Figure 3, b=9 and therefore $Z_{B}=51$ bits. It is easy to prove that always $Z_{B} \leq Z_{A}$. We know that, Maximum value of $f\left(\mathrm{~A}^{\mathrm{I}}, \mathrm{b}\right)=\mathrm{b}^{\mathrm{N}}-1$.

Total number of bits required to represent $f$ in binary is

$$
Z_{B}=\left\lceil\log _{2} b^{N}\right\rceil=\left\lceil N \times \log _{2} b\right\rceil
$$

Always,

$$
\left\lceil N \times \log _{2} b\right\rceil \leq \mathrm{N} \times\left\lceil\log _{2} b\right\rceil
$$

This verifies that $Z_{B} \leq Z_{A}$

\subsection{Formats Used for Encoding}

Original BS algorithm uses a block size of $3 \times 3$ for seg- 
mentation. There are three formats used by the original algorithm for encoding the sub-images.

Format1:

If $\mathrm{b} \in\{1,2 \ldots, 11\}$, then the coding format is

\begin{tabular}{|c|c|c|c|}
\hline $\mathrm{c}$ & $\mathrm{b}$ & $\mathrm{m}$ & Binary equivalent of $\mathrm{f}\left(\mathrm{A}^{\mathrm{I}}, \mathrm{b}\right)$ \\
1 bit & 7 bits & 8 bits & $\mathrm{Z}_{\mathrm{B}}$ bits \\
\hline
\end{tabular}

This format is economical when $b<2^{3.4}$

Format2:

If $\mathrm{b} \in(12,13, \ldots ., 128\}$, then the coding format is

\begin{tabular}{|c|c|c|c|c|}
\hline $\mathrm{c}$ & $\mathrm{b}$ & $\mathrm{m}(8$ & $\mathrm{P}(\min$, & $\begin{array}{c}\text { Binary equivalent of } \\
\left(g^{I} \text { for } 0 \leq i \leq 8,\right.\end{array}$ \\
$(1 \mathrm{bit})$ & $(7$ bits $)$ & bits $)$ & $\begin{array}{c}\text { max }) \\
(7 \text { bits })\end{array}$ & $\begin{array}{c}\left.i \neq i_{\min }, i \neq i_{\max }\right)_{b} \\
\left(\left\lceil\log _{2} b^{7}\right\rceil \text { bits }\right)\end{array}$ \\
\hline
\end{tabular}

Here $\mathrm{P}(\min , \max )$ is a pair of two 3 bit numbers indicating the position of minimum and maximum values. If $b>11$, writing the positions of minimum and maximum values is economical than coding them.

\section{Format 3:}

If $b \in(129,130, \ldots ., 256\}$, then the coding format is

\begin{tabular}{|c|c|}
\hline $\mathrm{c}$ & The original nine gray values: $\mathrm{g}_{0}, \mathrm{~g}_{1}, \ldots \mathrm{g}_{8}$ \\
$(1 \mathrm{bit})$ & $(72$ bits $)$ \\
\hline
\end{tabular}

Here, $\mathrm{c}$ stands for the category bit. If $\mathrm{c}$ is 0 , then the block is encoded using Formats 1 or 2; otherwise Format 3 is used.

\subsection{Hierarchical Use of BS Technique}

The encoded result of Subsection 2.1 can be compressed further in a hierarchical manner. We can imagine that there is a so-called "base-image", whose gray values are b0, b1, b2, .., b255; then, since it is a kind of image (except that each value is a base value of a sub-image rather than a gray value of a pixel), we can use the same BS technique to compress these base values. The details are omitted. Besides $b$, the minimal value $m$ of each block can also be grouped and compressed similarly. We can repeat the same procedure to encode $b$ and $m$ values further.

\section{PROPOSED METHOD}

In the proposed method, we made following modifications to the basic BS method.

1) Prediction

2) Increasing the block size from $3 \times 3$ to $4 \times 4$

3) All-zero block removal

4) Coding the minimum value and base value

\subsection{Prediction}

After reviewing the BS method, it is found that number of bits required for a sub-image is decided by the value of base ' $b$ '. If ' $b$ ' is reduced, the number of bits required for a sub-image is also reduced. In the proposed method, prediction is used to reduce the value of ' $b$ ' significantly. A predictor generates at each pixel of the input image the anticipated value of that pixel based on some of the past inputs. The output of the predictor is then rounded to the nearest integer, denoted $x_{n}$ and used to form the difference or prediction error

$$
e_{n}=x_{n}-\hat{x_{n}}
$$

This prediction error is coded by the proposed entropy coder. The decoder reconstructs $e_{n}$ from received code words and perform the reverse operation

$$
x_{n}=e_{n}+\hat{x_{n}}
$$

The quality of the prediction for each pixel directly affects how efficiently the prediction error can be encoded. The better this prediction is less is the information that must be carried by the prediction error signal. This, in turn, leads to fewer bits. One way to improve the prediction used for each pixel is to make a number of predictions then chose the one which comes closest to the actual value [21]. This method also called as switched prediction has the major disadvantage that the choice of prediction for each pixel must be sent as overhead information. The proposed prediction scheme uses two predictors and one of them is chosen for every block of pixels of size $4 \times 4$. Thus, the choice of prediction is to be made only once for the entire $4 \times 4$ block. This reduces the amount of overhead. The two predictions are given in Eq.16 and 17, in that, $\operatorname{Pr}_{1}$ is the popular MED predictor used in JPEG-LS standard and $\mathrm{Pr}_{2}$ is the one used by [5] for the compression of mammography images.

For the entire pixels of a block of size $4 \times 4$, one of the two predictions is chosen depending on the smoothness property of the predicted blocks. Here, smoothness is measured as the difference between maximum and minimum pixel values in the block. The predictor that gives the lowest difference value will be selected for that block. The advantage here is that the overhead required for each block is only one bit.

$$
\begin{aligned}
& =\min (\mathrm{A}, \mathrm{B}) \text { if } \mathrm{C} \geq \max (\mathrm{A}, \mathrm{B}) . \\
\operatorname{Pr}_{1} & =\max (\mathrm{A}, \mathrm{B}) \text { if } \mathrm{C}<=\min (\mathrm{A}, \mathrm{B}) . \\
& =\mathrm{A}+\mathrm{B}-\mathrm{C} \quad \text { otherwise. } \\
\mathrm{Pr}_{2}=\lceil(A \times 0.1 & +B \times 0.3+C \times 0.1+D \times 0.2+E \times 0.2+F \times 0.1)\rceil
\end{aligned}
$$

Here, A, B, C, D, E and F are the neighbors of pixel involved in prediction as depicted in Figure 4.

The proposed switched prediction is described in the equation form in 18 where $\mathrm{d} 1$ and $\mathrm{d} 2$ are the differ- 


\begin{tabular}{|l|l|l|l|l|}
\hline & & & & \\
\hline & C & B & E & F \\
\hline & A & X & & \\
\hline & D & & & \\
\hline & & & & \\
\hline
\end{tabular}

Figure 4. Neighbors of pixel involved in prediction.

ences between maximum and minimum values for the two blocks obtained using the two predictors $\operatorname{Pr}_{1}$ and $\operatorname{Pr}_{2}$ respectively.

$$
\operatorname{Pr} \quad \begin{aligned}
=\operatorname{Pr}_{1} \text { if } \mathrm{d} 1<\mathrm{d} 2 \\
\quad=\operatorname{Pr}_{2} \text { otherwise }
\end{aligned}
$$

Figure 5 illustrates the prediction technique. It shows two error images which are obtained by using two predictors $\operatorname{Pr}_{1}$ and $\operatorname{Pr}_{2}$ respectively. The BS algorithm divides them into $4 \times 4$ sub-images and computes the difference between maximum and minimum pixel values for all the four sub-images. For the first sub-image, difference ' $\mathrm{d} 1$ ' is 6 and ' $\mathrm{d} 2$ ' is 8 , where $\mathrm{d} 1$ and $\mathrm{d} 2$ are the differences of the sub-images corresponding to the predictors $\operatorname{Pr}_{1}$ and $\operatorname{Pr}_{2}$ respectively. Now, since $\mathrm{d} 1<\mathrm{d} 2$, the prediction $\operatorname{Pr}$ considers $4 \times 4$ sub-image of predictor $\mathrm{Pr}_{1}$ for further processing. This procedure is repeated for all the other sub-images. The resulting error image is shown in Figure 6. We use a separate file predict to store the choice of predictor made at every sub-image.

Error Image obtained

using prediction $\mathrm{p}$

\begin{tabular}{|c|c|c|c|c|c|c|c|}
\hline-1 & 0 & 2 & 0 & -2 & 3 & 2 & 1 \\
\hline 0 & 3 & 3 & 0 & 2 & 2 & 2 & 1 \\
\hline 2 & 3 & 4 & 0 & 2 & 0 & 1 & 4 \\
\hline 0 & -2 & 3 & -1 & 0 & 5 & -2 & -3 \\
\hline 0 & 0 & 0 & 0 & 2 & 3 & -1 & 0 \\
\hline-1 & 1 & 1 & 1 & 1 & 3 & -1 & 0 \\
\hline 0 & 2 & 1 & 0 & 0 & 3 & 2 & 0 \\
\hline-1 & 1 & 1 & 2 & 2 & 2 & 2 & 1 \\
\hline
\end{tabular}

Error Image obtained using prediction $\mathrm{p}_{\mathrm{r}}$

\begin{tabular}{|c|c|c|c|c|c|c|c|}
\hline-1 & 0 & 2 & 0 & -2 & 3 & 2 & 2 \\
\hline 0 & 3 & 3 & 0 & 2 & 2 & 2 & 1 \\
\hline 2 & 3 & 4 & 0 & 2 & 0 & 1 & 4 \\
\hline 0 & -4 & 3 & -1 & 0 & 3 & -2 & -3 \\
\hline 0 & 0 & 0 & 0 & 2 & 3 & -1 & 0 \\
\hline-1 & 1 & 1 & 1 & 1 & 3 & -1 & 0 \\
\hline 0 & 4 & 1 & 0 & 0 & 6 & 2 & 0 \\
\hline-1 & 1 & 1 & 2 & 2 & 2 & 2 & 1 \\
\hline
\end{tabular}

Figure 5. Prediction technique.

\subsection{Increasing the Block Size from $3 \times 3$ to $4 \times 4$}

It is obvious that smaller block size gives lower $b$ value but at the cost of increased overhead for the total image. The basic BST algorithm uses a block size of $3 \times 3$ to achieve optimum balance between amount of overhead and compression efficiency. Since the prediction increases smoothness, a larger block size can be chosen without significant difference in the smoothness. Certainly, this will improve the compression ratio. We have tested the proposed algorithm using different block sizes and found that block size of $4 \times 4$ gives the best result. This is supported by Table 1 giving the average compression ratios obtained for the 50 mammography images for various block sizes.

\subsection{All-Zero Block Removal}

Mammography images are highly correlated so that pixels inside most of the sub-images are same. During prediction and subtraction they have the highest chance of becoming zeros. If a sub-image of the error image has all its pixel values as zero, then that is marked as an all-zero block by storing a bit 1 in the encoding format. For each of the remaining sub-images, bit 0 is stored in the encoding format and is retained for further processing. Mammography images of MIAS data-set shows very large amount of all-zero blocks. This is supported by Table 2 showing average number of all-zero blocks present in the 50 mammography images of the MIAS dataset.

For these images, total bits required for marking the presence or absence of all-zero blocks is 65536 , since

\begin{tabular}{|l|l|l|l|l|l|l|l|}
\hline-1 & 0 & 2 & 0 & -2 & 3 & 2 & 2 \\
\hline 0 & 3 & 3 & 0 & 2 & 2 & 2 & 1 \\
\hline 2 & 3 & 4 & 0 & 2 & 0 & 1 & 4 \\
\hline 0 & -2 & 3 & -1 & 0 & 3 & -2 & -3 \\
\hline 0 & 0 & 0 & 0 & 2 & 3 & -1 & 0 \\
\hline-1 & 1 & 1 & 1 & 1 & 3 & -1 & 0 \\
\hline 0 & 2 & 1 & 0 & 0 & 3 & 2 & 0 \\
\hline-1 & 1 & 1 & 2 & 2 & 2 & 2 & 1 \\
\hline
\end{tabular}

Figure 6. Resulting error image.

Table 1. Average compression ratio obtained for various block sizes.

\begin{tabular}{ccccc}
\hline $3 \times 3$ & $4 \times 4$ & $4 \times 8$ & $8 \times 4$ & $8 \times 8$ \\
\hline 6.18 & $\mathbf{6 . 4 4}$ & 6.15 & 6.18 & 5.92 \\
\hline
\end{tabular}

Table 2. Average number of all-zero blocks present in the 50 MIAS images.

\begin{tabular}{cccc}
\hline Image size & $\begin{array}{c}\text { No. of blocks } \\
\text { of size } 4 \times 4\end{array}$ & $\begin{array}{c}\text { Average no. of } \\
\text { all-zero blocks }\end{array}$ & $\begin{array}{c}\text { Remain- } \\
\text { ing blocks }\end{array}$ \\
\hline $1024 \times 1024$ & 65536 & 32282 & 33254 \\
\hline
\end{tabular}


total sub-images are 65536 . The error image will have both negative and positive pixel values since the prediction has changed the range of the pixel values from $[0$, $255]$ to $[-255,255]$. Therefore, 9 bits are required to re- cord the pixel values. The approximate average compression ratio obtained by all-zero block removal for the 50 MIAS images considered in Table 2 can be estimated by the following formula.

$$
\begin{aligned}
C R & =\frac{1024 \times 1024 \times 8}{65536+\text { remaining blocks } \times \text { pixels per block } \times \text { bits per pixel used for coding }} \\
& =\frac{1024 \times 1024 \times 8}{65536+33254 \times 16 \times 9}=1.73 .
\end{aligned}
$$

Here the value 65536 indicates the overhead bits required for marking the presence or absence of all-zero blocks. The numerator gives total bits used by the original uncompressed image. This computation clearly shows that removal of all-zero blocks alone gives an approximate compression ratio of 1.73 .

\subsection{Coding the Minimum Value and the Base Value}

The error image will have both negative and positive pixel values. The prediction has changed the range of the minimum value from $[0,255]$ to $[-255,255]$. Therefore, 9 bits are required to record the minimum value. By studying various images, it is found that minimum values are ranging from -128 to 64 . More concentration is observed between -8 to 0 . Similarly, base values are concentrated in the range 1 to 15 . This statistics is supported by Table 3 of average number of minimum values and base values for the 50 mammography images.

To exploit this redundancy, we use a typical categorize and coding technique. A four bit code is used to identify the minimum values. Minimum values less than 1 and greater than -15 are given with codes 0 to 14 , whereas other minimum values are represented by the code 15 followed by their actual 9 bit values. A scheme similar to this can be used to code the base values also. Base values between 1 and 15 are identified by using 4 bit codes whereas values greater than 15 are identified using the code 15 followed by their actual 9 bit values. The two four bit codes for each of the sub-images are combined to get an 8 bit number. Such 8 bit numbers are stored in a file and Huffman encoded at the end.

\subsection{System Overview}

As shown in Figure 7, we first divide the error image

Table 3. Average number of minimum and base values in 50 MIAS images.

\begin{tabular}{ccc}
$\begin{array}{l}\text { Average No. of } \\
\text { minimum and } \\
\text { base values }\end{array}$ & $\begin{array}{l}\text { Average no. of mini- } \\
\text { mum values in the } \\
\text { range }-14 \text { to } 0\end{array}$ & $\begin{array}{l}\text { Average no. of } \\
\text { base values in } \\
\text { the range 1 to 15 }\end{array}$ \\
\hline 33253 & 32466 & 33097 \\
\hline
\end{tabular}

into sub-images of size $4 \times 4$. The sub-images are then processed one by one. For each sub-image, we have to determine whether it belongs to all-zero category and if so they are removed. The remaining blocks are retained for further processing.

The two binary files zero and predict are separately run length encoded, grouped and Huffman encoded. Also, the two four bit files min and base are combined to form an eight bit file and Huffman encoded.

\subsection{Decoding a Sub-Image}

Following are the decoding steps:

1) The files min, base, zero and predict are reconstructed.

2) The decoding algorithm first checks whether the block is an all-zero block or not. If all-zero, then a $4 \times 4$ block of zero's is generated.

3) Otherwise, base value and minimum value are first obtained by using the files min and base. The modified image $\mathrm{A}^{\mathrm{I}}$ is reconstructed as explained in Section 2 and $4 \times 4$ error image is obtained by adding min value to it.

4) Type of prediction used is read from the predict file. The prediction rule is applied to the $4 \times 4$ error image and the original $4 \times 4$ sub-image is reconstructed.

\section{RESULTS}

We evaluated the performance of the proposed scheme using the 50 gray-scale images of the MIAS dataset that include three varieties of images: Normal, Benign and Malignant. The MIAS is a European Society that researches mammograms and supplies over 11 countries with real world clinical data. Films taken from the UK national Breast Screening Program have been digitized to 50 micron pixel edge and representing each pixel with an 8 bit word. MATLAB is the tool used for simulation. All the simulation was conducted on a $1.7 \mathrm{GHZ}$ processor and was supplied with the same set of 50 mammography images. Each mammogram has a resolution of $1024 \times 1024$ and uses 8 bits/pixel. Results of the proposed method are compared with that of the popular methods. Comparison of Compression results for the 50 MIAS images is shown in Table 4. This set includes all the three varieties of images namely normal, benign and malignant. 


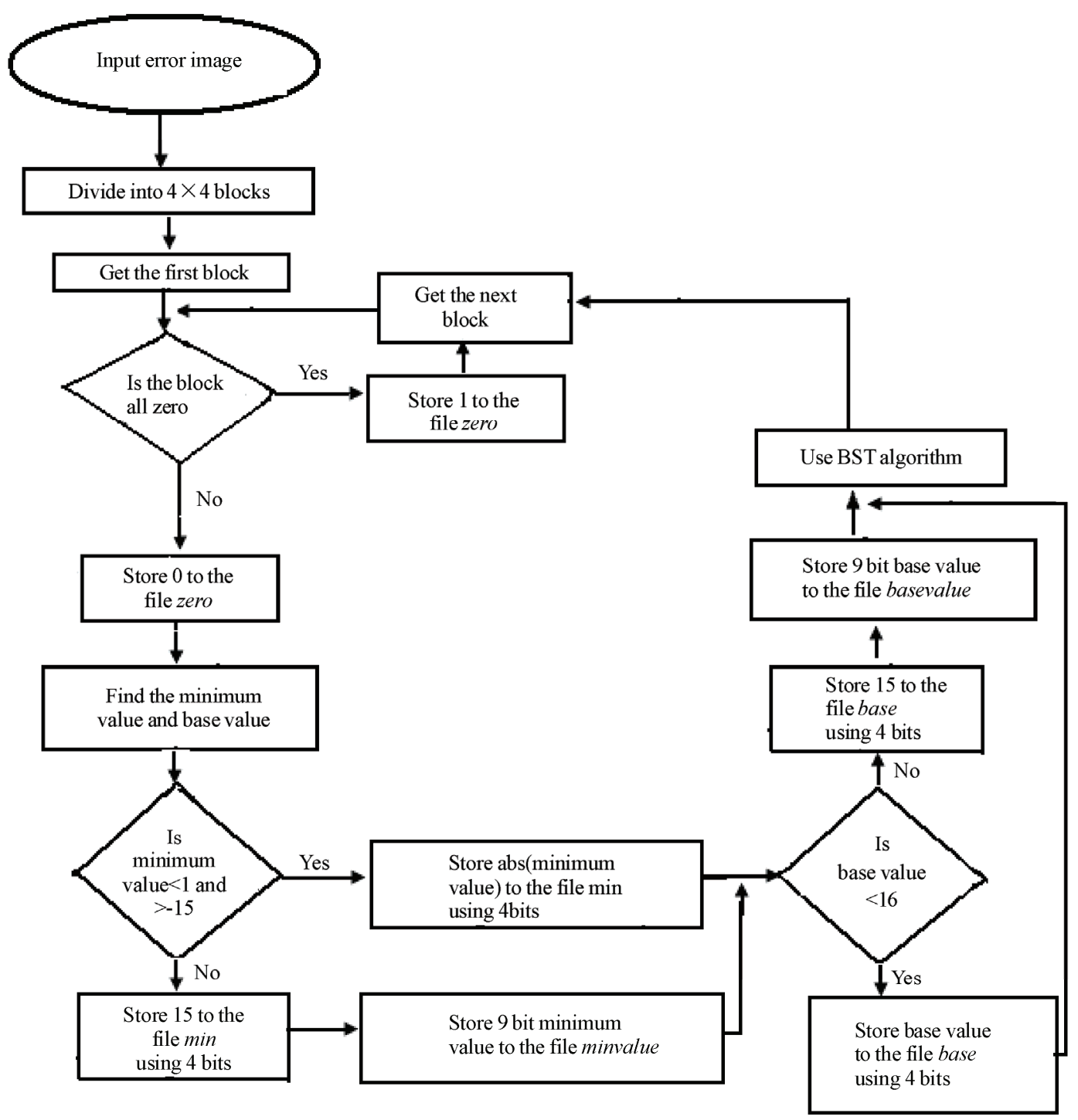

Figure 7. System overview.

Table 4. Comparison of compression results for the 50 MIAS images.

\begin{tabular}{ccccc}
\hline PNG & JBIG & JPEG2000 & JPEGLS & PROPOSED \\
\hline 4.30 & 5.88 & 6.29 & 6.39 & 6.44 \\
\hline
\end{tabular}

Figure 8 and Figure 9 show the two images mdb040. pgm and mdb025.pgm that gives best and the worst compression ratio respectively.

\section{CONCLUSIONS}

Several techniques have been used for the lossless compression of mammography images; none of them have used the smoothness property of the images. Our study has shown that there is very large number of zero blocks present in mammography images. We have picked up the concept of Base switching transformation and success- fully optimized and applied it in conjunction with other existing compression methods to digitized high resolution mammography images. Comparison with other approaches is given for a set of 50 high resolution digital mammograms comprising of normal, benign and malignant images. Compared with the PNG method, one of the international standards, JBIG, performs better by $36 \%$. Transformation method based JPEG200, another international compression standard, when used in lossless mode, performs slightly better than JBIG by $7 \%$. Whereas, the latest standard for lossless image compression JPEG-LS based on prediction based method, performs best among the four international standards of lossless coding techniques. It gives a compression ratio of 6.39 which is $1.5 \%$ better than the JPEG 2000 . Finally, for these images, the proposed method performs better than PNG, JBIG, JPEG2000 and JPEG-LS by 50\%, 9.5\%, 


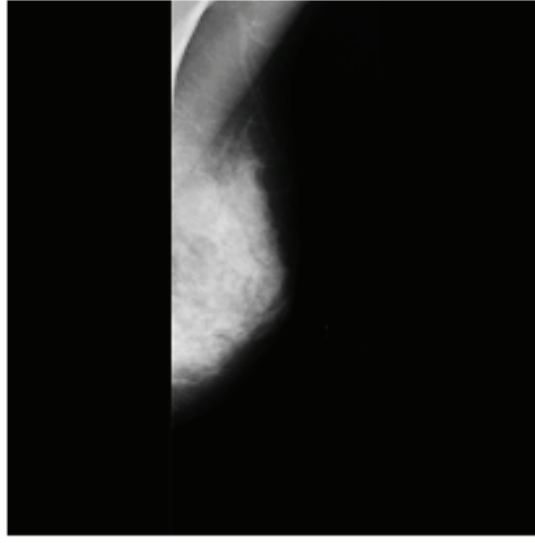

Figure 8. Image mdb040. $\mathrm{CR}=14.16$.

$2.4 \%$ and approximately $1 \%$ respectively. The success of our method is primarily due to its zero block removal procedure, compression of the overheads and the switched prediction used. It should be also noted that the speed of the BST method is very much comparable with the speed of other standard methods as verified by [18]. Further investigation on improvement of the performance of our method is under way, by developing more suitable prediction methods. Motivated by the results obtained here, our next study will carry out the compression of larger database of natural images and medical images obtained by other modalities.

\section{REFERENCES}

[1] Saxena, S., Rekhi, B., Bansal, A., Bagya, A., Chintamani, and Murthy, N. S., (2005) Clinico-morphological patterns of breast cancer including family history in a New Delhi Hospital, India - a cross sectional study, World Journal of Surgical Oncology, 1-8.

[2] Cahoon, T. C., Sulton, M. A, and Bezdek, J. C. (2000), Breast cancer detection using image processing techniques, The 9th IEEE International Conference on Fuzzy Systems, 2, 973-976.

[3] Penedo, M., Pearlman, W. A., Tahoces, P. G., Souto, M., and Vidal, J. J., (2003) Region-based wavelet coding methods for digital mammography, IEEE Transactions on Medical Imaging, 22, 1288-1296.

[4] Wu, X. L., (1997), Efficient lossless compression of Continuous-tone images via context selection and quantization, IEEE Transaction. on Image Processing, 6, 656664.

[5] Ratakonda, K. and Ahuja, N. (2002), Lossless image compression with multi-scale segmentation, IEEE Transactions on Image Processing, 11, 1228-1237.

[6] Weinberger, M. J., Rissanen, J., and Asps, R., (1996) Application of universal context modeling to lossless compression of gray scale images, IEEE Transaction on Image Processing, 5, 575-586.

[7] Grecos, C., Jiang, J., and Edirisinghe, E. A., (2001) Two Low cost algorithms for improved edge detection in

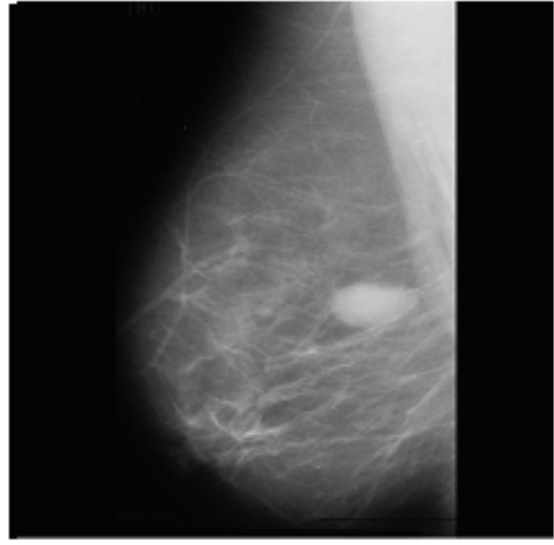

Figure 9. Image mdb025. $\mathrm{CR}=4.55$.

JPEG-LS, IEEE Transactions on Consumer Electronics, 47, 466-472.

[8] Weinberger, M. J., Seroussi, G., and Sapiro, G., (2000) The LOCO-I lossless image compression algorithm: Principles and standardization into JPEG-LS, IEEE Transactions on Image processing, 9, 1309-1324.

[9] Sung, M. M., Kim, H.-J., Kim, E.-K., Kwak, J.-Y., Kyung, J., and Yoo, H.-S., (2002) Clinical evaluation of JPEG 2000 compression for digital mammography, IEEE Transactions on Nuclear Science, 49, 827-832.

[10] Neekabadi, A., Samavi, S., and Karimi, N., (2007) Lossless compression of mammographic images by chronological sifting of prediction errors, IEEE Pacific Rim Conference on Communications, Computers \& Signal Processing, 58-61.

[11] Li, X., Krishnan, S., and Marwan, N. W., (2004) A novel way of lossless compression of digital mammograms using grammar codes, Canadian Conference on Electrical and Computer Engineering, 4, 2085-2088.

[12] da Silva, L. S. and Scharcanski, J., (2005) A lossless compression approach for mammographic digital images based on the delaunay triangulation, International Conference on Image Processing, 2, 758-761.

[13] Khademi, A. and Krishnan, S., (2005) Comparison of JPEG2000 and other lossless vompression dchemes for figital mammograms, Proceedings of the IEEE Engineering in Medicine and Biology Conference, 37713774.

[14] Shen, L. and Rangayyan, R. M., (1997) A segmentation based lossless image coding method for high-resolution medical image compression, IEEE Transactions on Medical imaging, 16, 301-307.

[15] Ranganathan, N., Romaniuk, S. G., and Namuduri, K. R., (1995) A lossless image compression algorithm using variable block segmentation, IEEE Transactions on Image Processing, 4, 1396-1406.

[16] Namuduri, K. R., Ranganathan, N., and Rashedi, H., (1996) SVBS: A high-resolution medical image compression algorithm using slicing with variable block size segmentation, IEEE Proceedings of ICPR, 919-923.

[17] Alsaiegh, M. Y. and Krishnan, S. (2001), Fixed blockbased lossless compression of digital mammograms, Canadian Conference on Electrical and Computer Engineering, 2, 937-942. 
[18] Chuang, T.-J. and Lin, J. C., (1998) A new algorithm for lossless still image compression, Pattern Recognition, 31, 1343-1352.

[19] Chang, C.-C., Hsieh, C.-P., and Hsiao, J.-Y., (2003) A new approach to lossless image compression, Proceedings of ICCT'03, 1734-38.

[20] Ravikumar, M. S., Koliwad, S., and Dwarakish, G. S.,
(2008) Lossless compression of digital mammography using fixed block segmentation and pixel grouping, Proceedings of IEEE $6^{\text {th }}$ Indian Conference on Computer Vision Graphics and Image Processing, 201-206.

[21] Sayood, K., (2003) Lossless compression handbook, First edition, Academic Press, USA, 207-223. 